\title{
“Underwater" endoscopic submucosal dissection: a novel technique for complete resection of a rectal neuroendocrine tumor
}

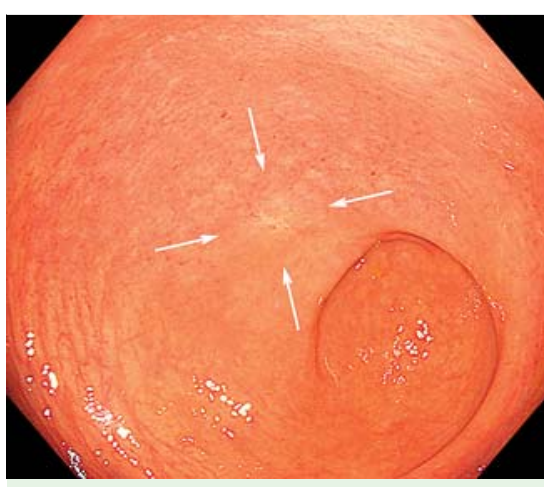

Fig. 1 Conventional endoscopic view of a poorly demarcated protruded lesion with a subepithelial aspect in the distal rectum (arrows).

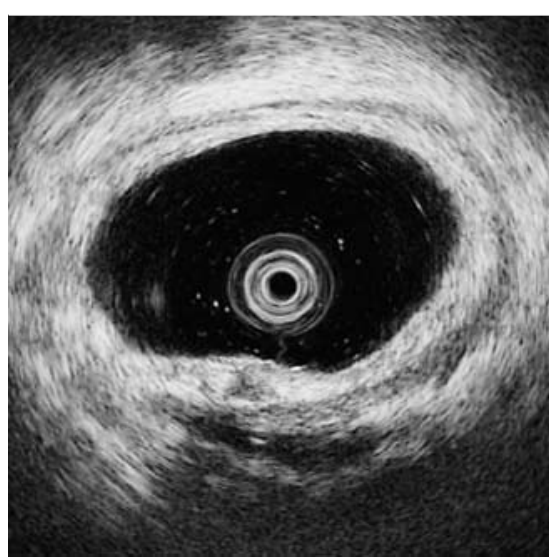

Fig. 2 An endoscopic ultrasonographic image of a rectal neuroendocrine tumor with a $20-\mathrm{MHz}$ miniature probe.

Many techniques for endoscopic resection of rectal neuroendocrine tumors (NETs) have been reported [1]. Among these, endoscopic submucosal dissection (ESD) has recently been applied [2]. Although ESD may have the advantage over conventional endoscopic mucosal resection of achieving a complete (R0) resection [3], the crush artifact caused by burn or coagulation from repeated exposure to electric current in this procedure cannot be avoided, and often results in non- $\mathrm{R} 0$ resection [4]. We present the case of a rectal NET resected using "underwater" ESD, a new method for complete resection without the creation of unnecessary burn artifacts ( $\bullet$ Video 1).

A 73-year-old man was diagnosed with a distal rectal NET by colonoscopy

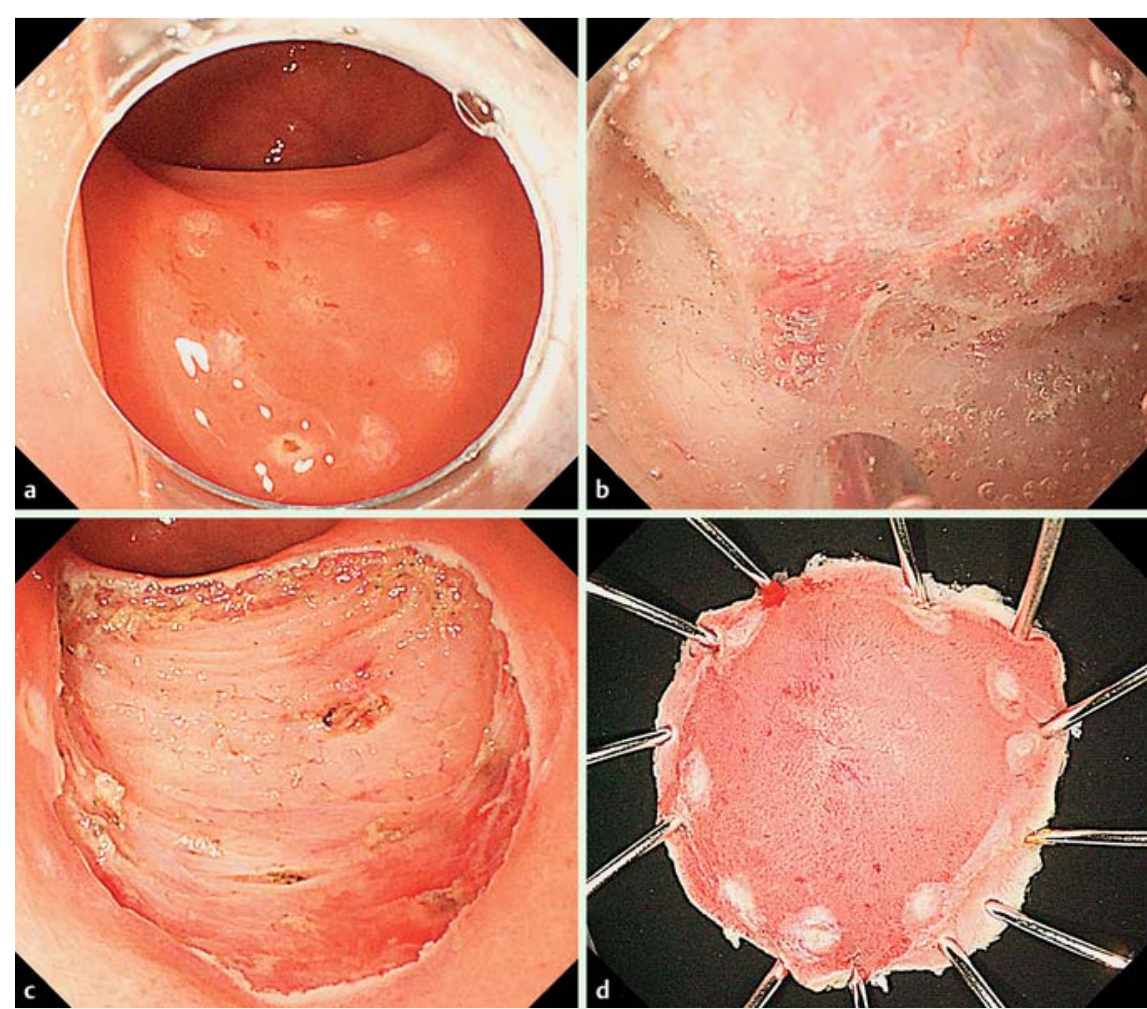

Fig. 3 The underwater endoscopic submucosal dissection (ESD) procedure. a The lateral margins of the tumor are marked with a coagulation current. $\mathbf{b}$ Underwater view of the dissection of the submucosal layer using a bipolar needle-knife (Jet B-knife, Zeon Medical Co., Tokyo, Japan) with an electrosurgical generator (VIO300D; Erbe Co., Tübingen, Germany). The swift coagulation mode was used for dissecting the submucosa, and the bipolar soft coagulation mode with a bipolar hemostatic forceps (H-S2518; Pentax Co., Tokyo, Japan) was used to control hemorrhage. c The ulcer bed after performing underwater ESD, with no apparent thermal damage to the muscle layer. $\mathbf{d}$ The resected specimen.

( Fig. 1). Endoscopic ultrasonography revealed that the tumor had invaded the deep submucosa, and that the submucosal margin between the tumor and the muscle layer was narrow and might be insufficient for R0 resection using the conventional endoscopic technique ( $\bullet$ Fig. 2 ).

A circumferential incision was made as for conventional ESD, and the rectal lumen was filled with saline. A bipolar needleknife (Jet B-knife; Zeon Medical Co., Tokyo, Japan) with swift coagulation mode was used to dissect the submucosa underwater. Although moderate bleeding occurred once during the submucosal dissection, this only required removal of the water to maintain a clear view and to use a hemostatic forceps; pre-coagulation underwater was possible against most

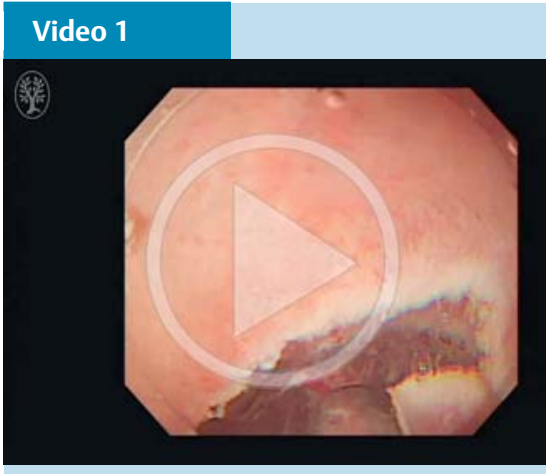

"Underwater" endoscopic submucosal dissection of a rectal neuroendocrine tumor. 

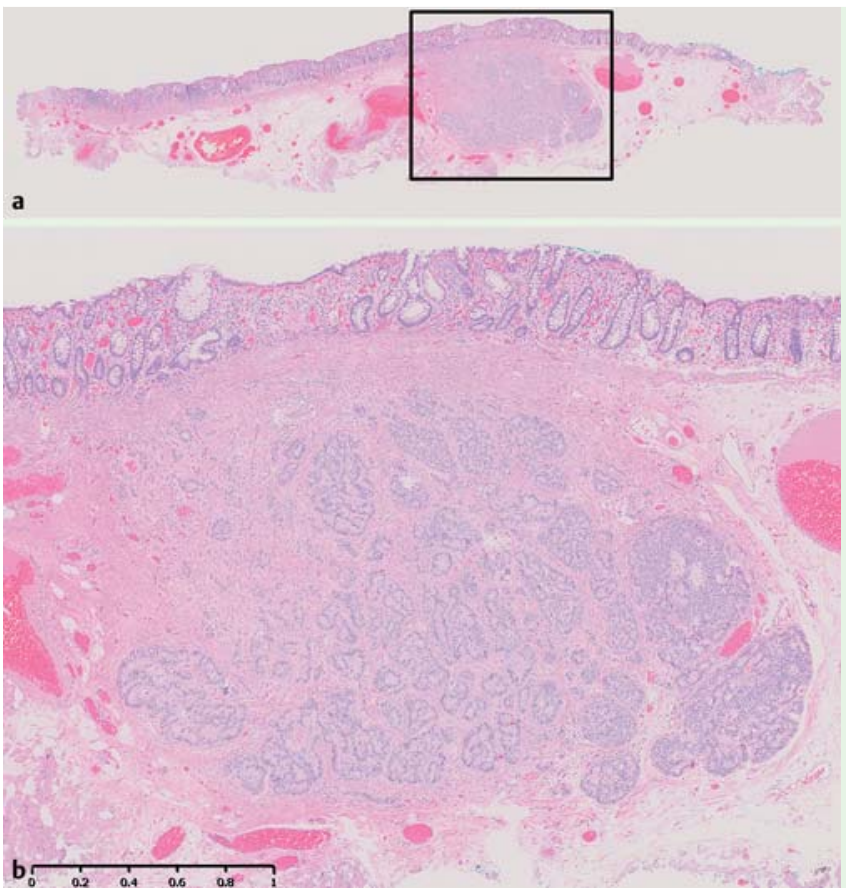

Fig. 4 The resected specimen was a welldifferentiated grade 1 neuroendocrine tumor invading the deep submucosal layer with tumor-free resection margins. a Loupe view (hematoxylin and eosin $[\mathrm{H} \& \mathrm{E}] \operatorname{stain} \times 5)$. $\mathbf{b}$ Highpower microscopic view (H\&E× 25).

vessels using the Jet B-knife with swift coagulation mode. Water was delivered via the waterjet function of the Jet B-knife when needed, and the lesion was precisely resected underwater, just above the muscle layer ( $\bullet$ Fig. 3 ).

Histological examination of the resected specimen revealed a grade 1 NET invading the deep submucosal layer with tumorfree resection margins ( $\bullet$ Fig. 4 ). The patient followed an uneventful clinical course.

For complete R0 resection of a rectal NET invading the deep submucosal layer endoscopically, the crush artifact associated with the electric current should be reduced. The "heat-sink" effect of water immersion can help to minimize the thermal damage to the resected specimen and muscle layer [5]. In addition, the "floating" effect of the mucosa and submucosa relative to the outer muscle layer provides good traction for resection, and the optical "zoom" effect of water immersion enables a more precise procedure [5]. Underwater ESD can therefore be a useful technique for $\mathrm{R} 0$ resection of rectal NETs.

Endoscopy_UCTN_Code_TTT_1AQ_2AD

Competing interests: None

\section{Shunsuke Yoshii ${ }^{1}$, Yoshito Hayashi ${ }^{1}$, Takahiro Matsui $^{2}$, Kenji Aoi ${ }^{1}$, Yoshiki Tsujii ${ }^{1}$, Hideki lijima', Tetsuo Takehara}

${ }^{1}$ Department of Gastroenterology and Hepatology, Osaka University Graduate School of Medicine, Osaka, Japan

2 Department of Pathology, Osaka University Graduate School of Medicine, Osaka, Japan

\section{References}

1 Kawaguti FS, de Oliveira JF, da Costa Martins $B$ et al. Underwater endoscopic resection of a neuroendocrine rectal tumor. Endoscopy 2015; 47 (Suppl. 01): E513-514

2 Lee DS, Jeon SW, Park SY et al. The feasibility of endoscopic submucosal dissection for rectal carcinoid tumors: comparison with endoscopic mucosal resection. Endoscopy 2010; 42: 647-651

3 Zhong DD, Shao LM, Cai JT. Endoscopic mucosal resection vs endoscopic submucosal dissection for rectal carcinoid tumours: a systematic review and meta-analysis. Colorectal Dis 2013; 15: 283-291

4 Chen $T$, Yao $L Q, X u M D$ et al. Efficacy and safety of endoscopic submucosal dissection for colorectal carcinoids. Clin Gastroenterol Hepatol 2015; pii: S1542-3565(15)01064-2. DOI: $10.1016 /$ j.cgh.2015.07.048

5 Binmoeller KF, Shah JN, Bhat YM et al. "Underwater" EMR of sporadic laterally spreading nonampullary duodenal adenomas (with video). Gastrointest Endosc 2013; 78: 496 502

\section{Bibliography}

Dol http://dx.doi.org/

10.1055/s-0042-101855

Endoscopy 2016; 48: E67-E68

(c) Georg Thieme Verlag KG

Stuttgart · New York

ISSN 0013-726X

\section{Corresponding author}

\section{Tetsuo Takehara, MD, PhD}

Department of Gastroenterology and Hepatology Osaka University Graduate School of Medicine

2-2, Yamadaoka suita

Osaka 565-0871

Japan

Fax: +81-6-68793629

takehara@gh.med.osaka-u.ac.jp 